\title{
ACCELERATION OF A FIXED POINT ALGORITHM FOR FLUID-STRUCTURE INTERACTION USING TRANSPIRATION CONDITIONS
}

\author{
Simone Deparis $^{1}$, Miguel Angel Fernández ${ }^{1}$ And Luca Formaggia ${ }^{2}$
}

\begin{abstract}
In this work, we address the numerical solution of fluid-structure interaction problems. This issue is particularly difficulty to tackle when the fluid and the solid densities are of the same order, for instance as it happens in hemodynamic applications, since fully implicit coupling schemes are required to ensure stability of the resulting method. Thus, at each time step, we have to solve a highly non-linear coupled system, since the fluid domain depends on the unknown displacement of the structure. Standard strategies for solving this non-linear problems, are fixed point based methods such as Block-Gauss-Seidel (BGS) iterations. Unfortunately, these methods are very CPU time consuming and usually show slow convergence. We propose a modified fixed-point algorithm which combines the standard BGS iterations with a transpiration formulation. Numerical experiments show the great improvement in computing time with respect to the standard BGS method.
\end{abstract}

Mathematics Subject Classification. 65M60, 65B99, 74F10.

\section{IntRoduction}

Large displacements low speed problems where a flexible elastic structure interacts with the flow of an external or internal fluid occur in many engineering fields: from civil engineering (aeroelasticity) to biomechanics (biomedical flows). One challenge arising in the numerical approximation of these fluid-structure problems is the definition of fast and accurate coupling algorithms that allow to predict the longterm time evolution maintaining the stability of the overall system. This issue is particularly difficult to face when the fluid and the solid densities are of the same order, for instance as it happens in hemodynamics, since only implicit schemes can ensure stability of the resulting method (see $[11,14,19]$ ). Thus, at each time step, the rule is to solve a highly coupled non-linear system (the fluid domain depends on the structural motion) using efficient methods that preserve, inside inner loops, the fluid-structure subsystem splitting. Standard strategies to solve this non-linear system are fixed-point based methods as Block-Jacobi or Block-Gauss-Seidel (BGS) iterations, see [3,14, 17, 19]. Recent advances concerns the use of Block Newton based method $[9,11,25]$ for a fast convergence towards the solution of the non-linear coupled problem.

In this paper we focus on accelerating numerical algorithms involving fixed-point iterations. It is well known that the standard Block-Jacobi or Block-Gauss-Seidel iterations are very CPU time consuming. Indeed, we must

\footnotetext{
Keywords and phrases. Fluid-structure interaction, Block-Gauss-Seidel iterations, transpiration, highly coupled non-linear problems, weak and strong coupling algorithms, partitioned procedures.

1 École Polytechnique Fédérale de Lausanne, IMA, 1015 Lausanne, Switzerland. e-mail: Simone.Deparis@epfl.ch

2 Politecnico di Milano, MOX, Piazza L. da Vinci 32, 20133 Milano, Italy.
} 


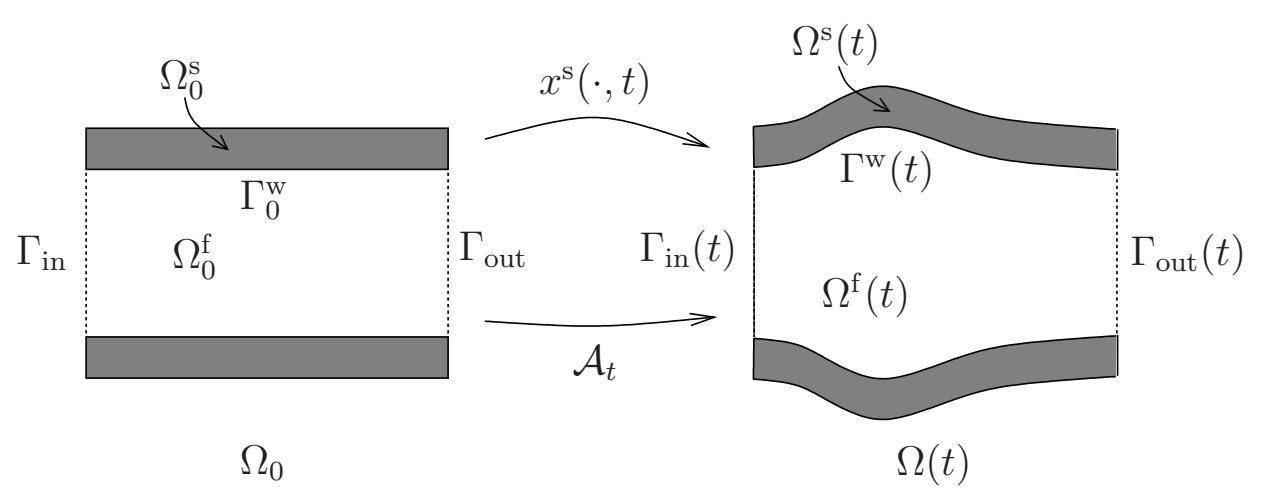

FiguRE 1. Geometric configurations.

add to the often slow convergence of the algorithms the cost of updating the fluid mesh, and the corresponding fluid matrices, at each iteration. We propose a modified fixed-point algorithm which combines the Block-GaussSeidel iterations (see [3]) with a transpiration formulation (see $[6,8,12,16,23,24]$ ). The underlying idea of our approach relies on the fact that standard BGS iterations associated to moderate interface deformations can be treated through transpiration techniques. These formulations do not require updating the fluid computational mesh and matrices. They only involve modifications of the interface boundary conditions.

The outline of this paper is as follows. In Section 2, we introduce the fluid-structure interaction problem and its mathematical description. We use the classical Arbitrary Lagrange-Euler formulation for the fluid. Section 3 we address the time discretization and the coupling algorithms, focusing on implicit schemes. We describe the BGS iterations. In Section 4 we provide the new algorithm, it combines the Block-Gauss-Seidel iterations with a transpiration formulation. Finally, in Section 5, we report the numerical results. They point out the great improvement in computing time of the proposed algorithm.

\section{Mechanical PROBlem}

The system under study occupies a moving domain $\Omega(t)$ in its actual configuration. It is made of a deformable structure $\Omega^{\mathrm{s}}(t)$ (vessel wall, pipe-line, ...) surrounding a fluid under motion (blood, oil, ...) in the complement $\Omega^{\mathrm{f}}(t)$ of $\Omega^{\mathrm{s}}(t)$ in $\Omega(t)$ (see Fig. 1). The problem consists in finding the time evolution of the configuration $\Omega^{\mathrm{f}}(t)$, as well as the velocity and Cauchy stress tensor within the fluid and the structure.

We assume the fluid to be Newtonian, viscous, homogeneous and incompressible. Its behavior is described by its velocity and pressure. The elastic solid under large displacements is described by its velocity and its stress tensor. The classical conservation laws of the continuum mechanics drive the evolution of these unknowns.

The fluid state satisfies the following incompressible Navier-Stokes equations, written in Eulerian conservative formulation,

$$
\begin{aligned}
\rho \frac{\partial u}{\partial t}+\operatorname{div}[\rho u \otimes u-\sigma(u, p)]=0, & \text { in } \quad \Omega^{\mathrm{f}}(t), \\
\operatorname{div} u=0, & \text { in } \quad \Omega^{\mathrm{f}}(t),
\end{aligned}
$$

where $u, p$ and $\rho$ stand for the fluid velocity, pressure and density, respectively. In addition, the fluid stress tensor is given by

$\mu$ being the fluid dynamic viscosity.

$$
\sigma(u, p)=-p \mathrm{I}+\mu\left[\nabla u+(\nabla u)^{\mathrm{T}}\right]
$$

Because of the large displacements, the time variation of the actual configuration cannot be neglected. The evolution of the fluid domain $\Omega^{\mathrm{f}}(t)$ is induced by the structural deformation through the fluid-structure 
interface $\Gamma^{\mathrm{w}}(t)$. Indeed, by definition

$$
\Omega^{\mathrm{f}}(t)=\Omega(t)-\bar{\Omega}^{\mathrm{s}}(t)
$$

It is convenient to describe $\Omega^{\mathrm{f}}(t)$ according to a map $\mathcal{A}_{t}$ acting in a fixed reference domain $\Omega_{0}^{\mathrm{f}}$. This approach is usually used for the solid domain $\Omega^{\mathrm{s}}(t)$, by means of the Lagrangian formulation [2, 13], as we will make precise later on. However, the choice of a Lagrangian mapping is in general not convenient for the fluid domain. Indeed, besides inducing large mesh deformations, it will be inconsistent with the inflow and outflow boundary definition (which are in general at a fixed spatial location). Therefore, the evolution of $\Omega^{\mathrm{f}}(t)$ is handled by introducing a family of homeomorphisms $\left\{\mathcal{A}_{t}\right\}_{t \in \mathbb{R}^{+}}$(see Fig. 1)

$$
\begin{array}{cccc}
\mathcal{A}_{t}: \Omega_{0}^{\mathrm{f}} & \longrightarrow & \mathbb{R}^{3} \\
x_{0} & \longmapsto & x=\mathcal{A}_{t}\left(x_{0}\right) .
\end{array}
$$

The choice of the configuration $\Omega_{0}^{\mathrm{f}}$ and of the map $\mathcal{A}_{t}$ is rather arbitrary, a part for the obvious requirement that $\mathcal{A}_{t}(\partial) \Omega_{0}^{\mathrm{f}}=\partial \Omega^{\mathrm{f}}(t), \partial \Omega^{\mathrm{f}}(t)$ being provided by the evolution of the fluid structure interface.

Hence the name of Arbitrary Lagrangian Eulerian (ALE) formulation given to the resulting equations. Given a material reference configuration $\Omega_{0}^{s}$ for the solid (see Fig. 1) the map

$$
x^{\mathrm{s}}: \Omega_{0}^{\mathrm{s}} \times \mathbb{R}^{+} \longrightarrow \mathbb{R}^{3},
$$

stands for the solid motion. For $x_{0} \in \Omega_{0}^{\mathrm{s}}, x^{\mathrm{s}}\left(x_{0}, t\right)$ represents the position at time $t \geq 0$ of the material point $x_{0}$. This corresponds to the classical Lagrangian flow $[2,13]$. The map $\mathcal{A}_{t}$ can be defined from $x_{\mid \Gamma_{0}^{\mathrm{s}}}^{\mathrm{s}}$, as an arbitrary extension over the domain $\Omega_{0}^{\mathrm{f}}$. In short, the ALE map $\mathcal{A}_{t}$ is given by

$$
\mathcal{A}_{t}\left(x_{0}\right)=\operatorname{Ext}\left(x^{\mathrm{s}}{ }_{\mid \Gamma_{0}^{\mathrm{w}}}^{\mathrm{w}}\right)\left(x_{0}, t\right), \quad \forall x_{0} \in \Omega_{0}^{\mathrm{f}},
$$

where Ext represents an extension operator from $\Gamma_{0}^{\mathrm{w}}$ to $\Omega_{0}^{\mathrm{f}}$.

Remark 2.1. In the sequel $\left.\frac{\partial}{\partial t}\right|_{x_{0}}$ stand for the time derivative operator keeping the space variable $x_{0}$ fixed. More formally, if $u: \Omega^{\mathrm{f}}(t) \times \mathbb{R}^{+} \rightarrow \mathbb{R}$, then

$$
\frac{\partial u}{\partial t}_{\mid x_{0}}=\left[\frac{\partial}{\partial t}\left(u \circ \mathcal{A}_{t}\right)\right] \circ \mathcal{A}_{t}^{-1}
$$

where the composition operator applies to the space variables only.

Remark 2.2. The operator Ext is arbitrarily defined inside $\Omega_{0}^{\mathrm{f}}$. It can be any reasonable extension of the material interface deformation:

$$
\mathcal{A}_{t}=\operatorname{Ext}\left(x_{\mid \Gamma_{0}^{\mathrm{w}}}^{\mathrm{s}}\right),\left.\quad \frac{\partial \mathcal{A}_{t}}{\partial t}\right|_{\Gamma_{0}^{\mathrm{w}}}=\dot{x}_{\mid \Gamma_{0}^{\mathrm{w}}}^{\mathrm{s}}
$$

For instance, Ext may be defined from an harmonic extension of the interface displacement.

This map allows us to rewrite the time derivative in (1), yielding to the classical incompressible Navier-Stokes equations written in ALE conservative formulation $[5,14]$, satisfied by $u: \Omega_{0}^{\mathrm{f}} \times \mathbb{R}^{+} \longrightarrow \mathbb{R}^{3}$ and $p: \Omega_{0}^{\mathrm{f}} \times \mathbb{R}^{+} \longrightarrow \mathbb{R}$

$$
\begin{aligned}
& \rho{\frac{\partial J_{\mathcal{A}_{t}} u}{\partial t}}_{\mid x_{0}}+J_{\mathcal{A}_{t}} \operatorname{div}[\rho u \otimes(u-w)-\sigma(u, p)]=0, \quad \text { in } \quad \Omega^{\mathrm{f}}(t), \\
& \operatorname{div} u=0, \quad \text { in } \Omega^{\mathrm{f}}(t),
\end{aligned}
$$

where the quantities $J_{\mathcal{A}_{t}}$ and $w$ are defined by:

$$
F_{\mathcal{A}_{t}}=\frac{\partial \mathcal{A}_{t}}{\partial x_{0}}, \quad J_{\mathcal{A}_{t}}=\operatorname{det}\left(F_{\mathcal{A}_{t}}\right)>0, \quad w=\frac{\partial \mathcal{A}_{t}}{\partial t} .
$$


Remark 2.3. By definition, $\dot{x}^{\text {s }}$ represents the solid velocity, whereas $w$ stands for the velocity of the fluid domain, which usually differs from the fluid velocity inside $\Omega^{\mathrm{f}}(t)$.

The solid evolution is given by its displacement $d$ around the reference material configuration $\Omega_{0}^{\mathrm{s}}$,

$$
x^{\mathrm{s}}\left(x_{0}, t\right)=x_{0}+d\left(x_{0}, t\right), \quad \forall x_{0} \in \Omega_{0}^{\mathrm{s}},
$$

and the stress tensor field $S$ (second Piola-Kirchoff tensor [2]). The field $S$ is related to $d$ through an appropriate constitutive law (see $[2,13])$. Then, the pair $(d, S)$ satisfies the non-linear elastodynamic equations [2]:

$$
\ddot{d}-\operatorname{div}_{0}(F S)=f, \quad \text { in } \quad \Omega_{0}^{\mathrm{s}}
$$

The coupling between the solid and the fluid is realized through standard boundary conditions at the fluidstructure interface $\Gamma_{0}^{\mathrm{w}}$, namely, the kinematic continuity of the velocity and the dynamic continuity of the stress [14]:

$$
\begin{aligned}
& u=w, \quad \text { on } \quad \Gamma_{0}^{\mathrm{w}}, \\
& F S n_{0}=J \sigma(u, p) F^{-\mathrm{T}} n_{0}, \quad \text { on } \quad \Gamma_{0}^{\mathrm{w}} \text {, }
\end{aligned}
$$

where $J$ and $F$ are defined by

$$
F=\frac{\partial x^{\mathrm{s}}}{\partial x_{0}}, \quad J=\operatorname{det}(F)>0
$$

and $n_{0}$ stands for the unit normal vector on $\Gamma_{0}^{\mathrm{w}}$ pointing inside $\Omega_{0}^{\mathrm{s}}$. In summary, the strong coupled problem, with a ALE formulation for the fluid, is given by:

$$
\begin{aligned}
& \rho{\left.\frac{\partial J_{\mathcal{A}_{t}} u}{\partial t}\right|_{x_{0}}+J_{\mathcal{A}_{t}} \operatorname{div}[\rho u \otimes(u-w)-\sigma(u, p)]=0,} \text { in } \quad \Omega^{\mathrm{f}}(t), \\
& \operatorname{div} u=0, \text { in } \Omega^{\mathrm{f}}(t), \\
& u=w, \text { on } \Gamma_{0}^{\mathrm{w}}, \\
& \sigma(u, p) n=g \text { on } \Gamma_{\text {in }}(t) \cup \Gamma_{\text {out }}(t), \\
& \ddot{d}-\operatorname{div}_{0}(F S)=f, \text { in } \quad \Omega_{0}^{\mathrm{s}}, \\
& F S n_{0}=J \sigma F^{-T} n_{0}, \text { on } \Gamma_{0}^{\mathrm{w}}, \\
& d=0, \text { on } \Gamma_{0}^{\mathrm{D}}, \\
& F S n_{0}=0, \text { on } \Gamma_{0}^{\mathrm{N}}, \\
& w=\frac{\partial \mathcal{A}_{t}}{\partial t}, \text { in } \quad \Omega_{0}^{\mathrm{f}},
\end{aligned}
$$

where $g$ stands for the external forces acting on the fluid. For simplification purposes, no fluid body forces are considered. The boundary conditions to be imposed on $\Gamma_{\text {in }}(t) \cup \Gamma_{\text {out }}(t)$ as well as on $\partial \Omega_{0}^{\mathrm{s}}-\Gamma_{0}^{\mathrm{w}}=\Gamma_{0}^{\mathrm{D}} \cup \Gamma_{0}^{\mathrm{N}}$ will depend on the problem under consideration. 
Problem (5) can be written in weak variational form as follows: find $\mathcal{A}_{t}: \Omega_{0}^{\mathrm{f}} \longrightarrow \mathbb{R}^{3}$, u: $\Omega^{\mathrm{f}}(t) \times \mathbb{R}^{+} \longrightarrow \mathbb{R}^{3}$, $p: \Omega^{\mathrm{f}}(t) \times \mathbb{R}^{+} \longrightarrow \mathbb{R}$ and $d: \Omega_{0}^{\mathrm{s}} \times \mathbb{R}^{+} \longrightarrow \mathbb{R}^{3}$ such that

$$
\begin{aligned}
& \rho \frac{\mathrm{d}}{\mathrm{d} t} \int_{\Omega^{\mathrm{f}}(t)} u \cdot v^{\mathrm{f}} \mathrm{d} x-\rho \int_{\Omega^{\mathrm{f}}(t)} \operatorname{div}[u \otimes(u-w)] \cdot v^{\mathrm{f}} \mathrm{d} x+\int_{\Omega^{\mathrm{f}}(t)} \sigma(u, p): \nabla v^{\mathrm{f}} \mathrm{d} x \\
& -\int_{\Gamma_{\text {in }}(t) \cup \Gamma_{\text {out }}(t)} g(t) \cdot v^{\mathrm{f}} \mathrm{d} a+\int_{\Omega^{\mathrm{f}}(t)} q^{\mathrm{f}} \operatorname{div} u \mathrm{~d} x=0, \quad \forall\left(v^{\mathrm{f}}, q^{\mathrm{f}}\right) \in V^{\mathrm{f}} \times Q^{\mathrm{f}}, \\
& u=w, \quad \text { on } \quad \Gamma_{0}^{\mathrm{w}}, \\
& m^{\mathrm{s}}\left(\ddot{d}, v^{\mathrm{s}}\right)+a^{\mathrm{s}}\left(d, v^{\mathrm{s}}\right)=\int_{\Omega_{0}^{\mathrm{s}}} f \cdot v^{\mathrm{s}} \mathrm{d} x-\int_{\Gamma_{0}^{\mathrm{w}}} J \sigma(u, p) F^{-\mathrm{T}} n_{0} \cdot v^{\mathrm{s}} \mathrm{d} x, \\
& \forall v \in V^{\mathrm{s}}=H_{\Gamma_{0}^{\mathrm{D}}}^{1}\left(\Omega_{0}^{\mathrm{s}}\right)^{3}, \\
& \mathcal{A}_{t}=\operatorname{Ext}\left(x_{\mid \Gamma_{0}^{\mathrm{w}}}^{\mathrm{s}}\right), \quad w=\frac{\partial \mathcal{A}_{t}}{\partial t}, \quad \text { in } \quad \Omega_{0}^{\mathrm{f}} .
\end{aligned}
$$

Here, the spaces of fluid test functions are defined, as in [10], by

$$
V^{\mathrm{f}}=\left\{v \circ \mathcal{A}_{t}^{-1}, \quad v \in H^{1}\left(\Omega_{0}^{\mathrm{f}}\right)^{3}\right\}, \quad Q^{\mathrm{f}}=\left\{q \circ \mathcal{A}_{t}^{-1}, \quad q \in L^{2}\left(\Omega_{0}^{\mathrm{f}}\right)\right\}
$$

and

$$
m^{\mathrm{s}}\left(\ddot{d}, v^{\mathrm{s}}\right)=\int_{\Omega_{0}^{\mathrm{s}}} \rho_{0} \ddot{d} \cdot v^{\mathrm{s}} \mathrm{d} x, \quad a^{\mathrm{s}}\left(d, v^{\mathrm{s}}\right)=\int_{\Omega_{0}^{\mathrm{s}}} F S: \nabla v^{\mathrm{s}} \mathrm{d} x,
$$

stand for the solid mass and stiffness integrals. Similar integrals appear when dealing with more general structures such as elastic beams or shells in large displacements (see $[1,14])$.

\section{Time Discretization: COUPling Algorithms}

Concerning the time discretization of (6) several schemes can be considered. A first strategy, for instance, leads to the standard loosely coupled algorithms [7,20,21]. It consists in using an explicit scheme for the fluid (respectively for the structure) and an implicit scheme for the structure (respectively for the fluid). Thus, at each time step, the fluid solution is completely determined starting from the solution of the previous time step and, once the fluid load at the interface has been computed, the structure can be advanced on time updating the position of the interface. In short, the geometry and the interface coupling are treated explicitly. This strategy is computationally cheap and performs well in many practical situations, for example, in aeroelasticity applications $[7,20,21]$. However, numerical experiments and some analysis on simplified models (see $[11,14,19])$ indicate that these staggered algorithms are unstable when the structure is light, more precisely when the fluid and structure densities are comparable, as it happens in hemodynamic applications. In these situations, fluidstructure equilibrium must be ensured at each time accurately. In other words, the geometry and the interface coupling have to be treated implicitly, and then implicit coupling schemes must be considered.

For these reasons, in this paper we will focus on fully coupled implicit schemes. In the sequel, we consider in (6) an implicit Euler treatment of the velocity derivatives in the fluid domain and a mid-point rule for the structural equation. The resulting time discretized problem writes: for $n=0,1, \ldots$, find $u^{n+1}: \Omega^{\mathrm{f}}\left(t_{n+1}\right) \longrightarrow \mathbb{R}^{3}$, 
$p^{n+1}: \Omega^{\mathrm{f}}\left(t_{n+1}\right) \longrightarrow \mathbb{R}$ and $d^{n+1}: \Omega_{0}^{\mathrm{s}} \longrightarrow \mathbb{R}^{3}$ satisfying the following coupled non-linear problem:

$$
\begin{aligned}
& \mathcal{A}_{t_{n+1}}=\operatorname{Ext}\left(x^{\mathrm{s}, n+1}{ }_{\mid \Gamma_{0}^{\mathrm{w}}}\right), \quad w^{n+1}=\frac{1}{\Delta t}\left(\mathcal{A}_{t_{n+1}}-\mathcal{A}_{t_{n}}\right), \quad \text { in } \quad \Omega_{0}^{\mathrm{f}}, \\
& \frac{\rho}{\Delta t} \int_{\Omega^{\mathrm{f}}\left(t_{n+1}\right)} u^{n+1} \cdot v^{\mathrm{f}} \mathrm{d} x+\rho \int_{\Omega^{\mathrm{f}}\left(t_{n+1}\right)} \operatorname{div}\left[u^{n+1} \otimes\left(u^{n+1}-w^{n+1}\right)\right] \cdot v^{\mathrm{f}} \mathrm{d} x \\
& +\int_{\Omega^{\mathrm{f}}\left(t_{n+1}\right)} \sigma\left(u^{n+1}, p^{n+1}\right): \nabla v^{\mathrm{f}} \mathrm{d} x-\int_{\Gamma_{\text {in }}\left(t_{n+1}\right) \cup \Gamma_{\text {out }}\left(t_{n+1}\right)} g\left(t_{n+1}\right) \cdot v^{\mathrm{f}} \mathrm{d} a \\
& +\int_{\Omega^{\mathrm{f}}\left(t_{n+1}\right)} q^{\mathrm{f}} \operatorname{div} u^{n+1} \mathrm{~d} x=\frac{\rho}{\Delta t} \int_{\Omega^{\mathrm{f}}\left(t_{n}\right)} u^{n} \cdot v^{\mathrm{f}} \mathrm{d} x, \quad \forall\left(v^{\mathrm{f}}, q^{\mathrm{f}}\right) \in V^{\mathrm{f}} \times Q^{\mathrm{f}}, \\
& u^{n+1}=w^{n+1}, \quad \text { on } \quad \Gamma^{\mathrm{w}}\left(t_{n+1}\right), \\
& \frac{1}{\Delta t} m^{\mathrm{s}}\left(\dot{d}^{n+1}-\dot{d}^{n}, v^{\mathrm{s}}\right)+\frac{1}{2}\left(a^{\mathrm{s}}\left(d^{n+1}, v^{\mathrm{s}}\right)+a^{\mathrm{s}}\left(d^{n}, v^{\mathrm{s}}\right)\right) \\
& =-\int_{\Gamma^{\mathrm{w}}\left(t_{n+1}\right)} \sigma\left(u^{n+1}, p^{n+1}\right) n \cdot v^{\mathrm{s}} \mathrm{d} a, \quad \forall v^{\mathrm{s}} \in V^{\mathrm{s}}, \\
& \frac{1}{2}\left(\dot{d}^{n+1}+\dot{d}^{n}\right)=\frac{1}{\Delta t}\left(d^{n+1}-d^{n}\right), \quad \text { in } \quad \Omega_{0}^{\mathrm{s}} .
\end{aligned}
$$

The values at $n=0$ are provided by the initial conditions.

Therefore, at each time step, we have to solve a highly non-linear system, where the fluid domain and the structural displacement are tightly coupled. Assuming that problem (7) has been appropriately discretized in space, for instance by a FEM formulation, we formally write the resulting non-linear system as a fixed point problem,

$$
\begin{aligned}
\left(\mathrm{x}^{\mathrm{f}}, \mathrm{w}^{\mathrm{f}}\right) & =\mathcal{M}\left(\left(\mathrm{x}^{\mathrm{f}}, \mathrm{w}^{\mathrm{f}}\right),\left(\mathrm{x}^{\mathrm{s}}, \mathrm{w}^{\mathrm{s}}\right)\right), & & \text { (fluid mesh update) }, \\
(\mathrm{u}, \mathrm{p}) & =\mathcal{F}\left((\mathrm{u}, \mathrm{p}),\left(\mathrm{x}^{\mathrm{f}}, \mathrm{w}^{\mathrm{f}}\right)\right), & & \text { (fluid subproblem), } \\
\left(\mathrm{x}^{\mathrm{s}}, \mathrm{w}^{\mathrm{s}}\right) & =\mathcal{S}\left(\left(\mathrm{x}^{\mathrm{s}}, \mathrm{w}^{\mathrm{s}}\right),(\mathrm{u}, \mathrm{p})\right), & & (\text { solid subproblem }),
\end{aligned}
$$

where $(\mathrm{u}, \mathrm{p}),\left(\mathrm{x}^{\mathrm{s}}, \mathrm{w}^{\mathrm{s}}\right)$ and $\left(\mathrm{x}^{\mathrm{f}}, \mathrm{w}^{\mathrm{f}}\right)$ stand, respectively, for the discretized fluid velocity and pressure, the structural motion and velocity, and the fluid domain motion and velocity at a given time step.

Standard strategies to solve (8) are Block-Jacobi or Block-Gauss-Seidel iterations, see [3]. For instance, the Block-Gauss-Seidel method consists in sub-iterating between the three subproblems. For $k \geq 0$ we set

$$
\begin{aligned}
& \left(\mathrm{x}_{k+1}^{\mathrm{f}}, \mathrm{w}_{k+1}^{\mathrm{f}}\right)=\mathcal{M}\left(\left(\mathrm{x}_{k}^{\mathrm{f}}, \mathrm{w}_{k}^{\mathrm{f}}\right),\left(\mathrm{x}_{k}^{\mathrm{s}}, \mathrm{w}_{k}^{\mathrm{s}}\right)\right), \quad \text { (update the fluid mesh), } \\
& \left(\mathrm{u}_{k+1}, \mathrm{p}_{k+1}\right)=\mathcal{F}\left(\left(\mathrm{u}_{k}, \mathrm{p}_{k}\right),\left(\mathrm{x}_{k+1}^{\mathrm{f}}, \mathrm{w}_{k+1}^{\mathrm{f}}\right)\right), \quad \text { (solve the fluid subproblem), } \\
& \left(\mathrm{x}_{k+1}^{\mathrm{s}}, \mathrm{w}_{k+1}^{\mathrm{s}}\right)=\mathcal{S}\left(\left(\mathrm{x}_{k}^{\mathrm{s}}, \mathrm{w}_{k}^{\mathrm{s}}\right),\left(\mathrm{u}_{k+1}, \mathrm{p}_{k+1}\right)\right), \quad \text { (solve the solid subproblem), }
\end{aligned}
$$

with $\mathrm{u}_{0}, \mathrm{p}_{0}, \mathrm{x}_{0}^{\mathrm{s}}, \mathrm{w}_{0}^{\mathrm{s}}, \mathrm{x}_{0}^{\mathrm{f}}$ and $\mathrm{w}_{0}^{\mathrm{f}}$ given from fluid-structure state computed at the previous time step. We stop the iterations when the difference between two successive interface displacements falls below a fixed tolerance (see also Sect. 4). In some cases, it is mandatory - in order to obtain convergence - to add a relaxation step to the structure displacement, see $[11,14,17,19]$.

It is well known that this strategy is very CPU time consuming. Indeed, we must add to the often slow convergence of the algorithm the cost of updating the fluid mesh and the corresponding fluid matrices at each iteration. In this paper, we propose a method to improve the performance of the standard BGS iteration (9). 


\section{New ALGorithm: BGS With transpiration}

Each iteration of the Block-Gauss-Seidel method (9) involves the numerical solution of the following non-

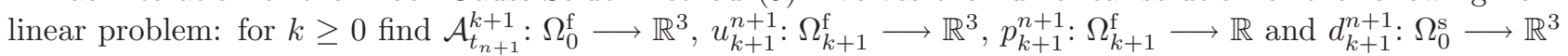
satisfying:

$$
\begin{aligned}
& \mathcal{A}_{t_{n+1}}^{k+1}=\operatorname{Ext}\left(x_{k}^{\mathrm{s}, n+1}{ }_{\mid \Gamma_{0}^{\mathrm{w}}}^{\mathrm{w}}\right), \quad w_{k+1}^{n+1}=\frac{1}{\Delta t}\left(\mathcal{A}_{t_{n+1}}^{k+1}-\mathcal{A}_{t_{n}}\right), \quad \text { in } \quad \Omega_{0}^{\mathrm{f}}, \\
& \frac{\rho}{\Delta t} \int_{\Omega_{k+1}^{\mathrm{f}}} u_{k+1}^{n+1} \cdot v^{\mathrm{f}} \mathrm{d} x+\rho \int_{\Omega_{k+1}^{\mathrm{f}}} \operatorname{div}\left[u_{k+1}^{n+1} \otimes\left(u_{k}^{n+1}-w_{k+1}^{n+1}\right)\right] \cdot v^{\mathrm{f}} \mathrm{d} x \\
& +\int_{\Omega_{k+1}^{\mathrm{f}}} \sigma\left(u_{k+1}^{n+1}, p_{k+1}^{n+1}\right): \nabla v^{\mathrm{f}} \mathrm{d} x-\int_{\Gamma_{\mathrm{in}} \cup \Gamma_{\text {out }}} g\left(t_{n+1}\right) \cdot v^{\mathrm{f}} \mathrm{d} a \\
& +\int_{\Omega_{k+1}^{\mathrm{f}}} q^{\mathrm{f}} \operatorname{div} u_{k+1}^{n+1} \mathrm{~d} x=\frac{\rho}{\Delta t} \int_{\Omega^{\mathrm{f}}\left(t_{n}\right)} u^{n} \cdot v^{\mathrm{f}} \mathrm{d} x, \quad \forall\left(v^{\mathrm{f}}, q^{\mathrm{f}}\right) \in V^{\mathrm{f}} \times Q^{\mathrm{f}}, \\
& u_{k+1}^{n+1}=w_{k+1}^{n+1} \text {, on } \Gamma_{k}^{\mathrm{w}} \text {, } \\
& \frac{1}{\Delta t} m^{\mathrm{s}}\left(\dot{d}_{k+1}^{n+1}-\dot{d}^{n}, v^{\mathrm{s}}\right)+\frac{1}{2}\left(a^{\mathrm{s}}\left(d_{k}^{n+1}, v^{\mathrm{s}}\right)+a^{\mathrm{s}}\left(d^{n}, v^{\mathrm{s}}\right)\right) \\
& =-\int_{\Gamma_{k+1}^{\mathrm{w}}} \sigma\left(u_{k+1}^{n+1}, p_{k+1}^{n+1}\right) n \cdot v^{\mathrm{s}} \mathrm{d} a, \quad \forall v^{\mathrm{s}} \in V^{\mathrm{s}}, \\
& \frac{1}{2}\left(\dot{d}_{k+1}^{n+1}+\dot{d}^{n}\right)=\frac{1}{\Delta t}\left(d_{k+1}^{n+1}-d^{n}\right), \quad \text { in } \quad \Omega_{0}^{\mathrm{s}} .
\end{aligned}
$$

Here $\mathcal{A}_{t_{n+1}}^{k+1}$ represents the ALE map computed at the $(k+1)$-th iteration of the BGS method, and $\Omega_{k+1}^{\mathrm{f}}$ the corresponding fluid domain, namely $\Omega_{k+1}^{\mathrm{f}}=\mathcal{A}_{t_{n+1}}^{k+1}\left(\Omega_{0}^{\mathrm{f}}\right)$.

Then main disadvantage of the standard BGS method lies on the fact that its iterations are very costly. Indeed, each iteration (10) involves an update of the fluid domain $\Omega_{k+1}^{\mathrm{f}}\left(t_{n+1}\right)$ and of its velocity $w_{k+1}^{n+1}$. Consequently, the fluid matrices have to be recomputed on this new configuration. This feature arises because we are using an ALE formulation for the fluid (large displacements are involved in the whole fluid-structure problem). However, between to successive BGS iterations the fluid-structure interface frequently exhibits moderate variations.

In order to be able to solve a low cost fluid-structure problems featuring moderate deformation, aeronautical engineers have developed transpiration techniques, from an idea of Lighthill [15]. These formulations do not require to update the computational grid, but only involve modifications of the interface boundary conditions (see for instance $[6,8,12,16,23,24])$. Consequently, whenever the fluid-structure interface shows a "small" variation (with respect to a specified tolerance $\mathrm{TOL}_{\text {trans }}$ ) between steps $k$ and $k+1$ of (10), the interface motion can be taken into account for the fluid problem through transpiration boundary conditions, without the need of updating the mesh and, consequently, the system matrices.

A transpiration interface condition on $\Gamma_{k}^{\mathrm{w}}$ can be derived, in a heuristic way (see $[12,16,23,24]$ and refer to $[6,8,18]$ for a more rigorous justification), from a truncated Taylor expansion of the fluid velocity in the neighborhood of the reference fluid-structure interface $\Gamma_{k}^{\mathrm{w}}$, see Figure 2:

$$
u_{k+1}^{n+1}\left(x_{k+1}^{\mathrm{f}}\right)=u_{k+1}^{n+1}\left(x_{k}^{\mathrm{f}}\right)-\nabla u_{k+1}^{n+1}\left(x_{k}^{\mathrm{f}}\right)\left(x_{k+1}^{\mathrm{f}}-x_{k}^{\mathrm{f}}\right)+o\left(\left\|x_{k+1}^{\mathrm{f}}-x_{k}^{\mathrm{f}}\right\|\right)
$$

on $\Gamma_{0}^{\mathrm{w}}$. Thus, from the kinematic condition

$$
u_{k+1}^{n+1}=w_{k+1}^{n+1}, \quad \text { on } \quad \Gamma_{k+1}^{\mathrm{w}},
$$




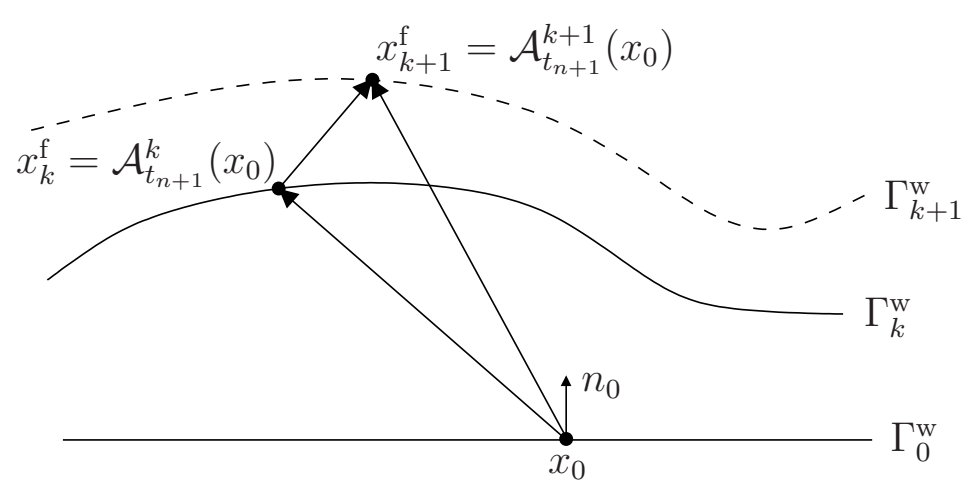

Figure 2. Taylor expansion of the fluid velocity.

we get the following transpiration condition on the known interface $\Gamma_{k}^{\mathrm{w}}$

$$
u_{k+1}^{n+1}=w_{k+1}^{n+1}-\nabla u_{k+1}^{n+1}\left(x_{k+1}^{\mathrm{f}}-x_{k}^{\mathrm{f}}\right), \quad \text { on } \quad \Gamma_{k}^{\mathrm{w}} .
$$

The implicit dependence on $u_{k+1}^{n+1}$ can be made explicit by modifying the relation into

$$
u_{k+1}^{n+1}=w_{k+1}^{n+1}-\nabla u_{k}^{n+1}\left(x_{k+1}^{\mathrm{f}}-x_{k}^{\mathrm{f}}\right)
$$

This latter condition can now be used to approximate the fluid subproblem in (10). Indeed, by setting

$$
\Omega_{\mathrm{trans}}^{\mathrm{f}}=\Omega_{k}^{\mathrm{f}}, \quad \Gamma_{\text {trans }}^{\mathrm{w}}=\Gamma_{k}^{\mathrm{w}}, \quad u_{\mathrm{trans}}^{n+1}=u_{k}^{n+1}, \quad w_{\text {trans }}^{n+1}=w_{k}^{n+1}
$$

we introduce the following transpired fluid subproblem

$$
\begin{aligned}
& w_{k+1}^{n+1}=\frac{1}{\Delta t}\left(d_{k}^{n+1}-d^{n}\right), \quad \text { on } \quad \Gamma_{0}^{\mathrm{w}}, \\
& \frac{\rho}{\Delta t} \int_{\Omega_{\text {trans }}^{\mathrm{f}}} u_{k+1}^{n+1} \cdot v^{\mathrm{f}} \mathrm{d} x+\rho \int_{\Omega_{\mathrm{trans}}^{\mathrm{f}}} \operatorname{div}\left[u_{k+1}^{n+1} \otimes\left(u_{\text {trans }}^{n+1}-w_{\text {trans }}^{n+1}\right)\right] \cdot v^{\mathrm{f}} \mathrm{d} x
\end{aligned}
$$

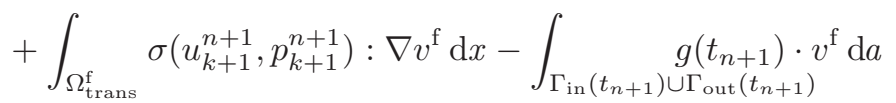

$$
\begin{aligned}
& +\int_{\Omega_{\text {trans }}^{\mathrm{f}}} q^{\mathrm{f}} \operatorname{div} u_{k+1}^{n+1} \mathrm{~d} x=\frac{\rho}{\Delta t} \int_{\Omega^{\mathrm{f}}\left(t_{n}\right)} u^{n} \cdot v^{\mathrm{f}} \mathrm{d} x, \quad \forall\left(v^{\mathrm{f}}, q^{\mathrm{f}}\right) \in V^{\mathrm{f}} \times Q^{\mathrm{f}}, \\
& u_{k+1}^{n+1}=w_{k+1}^{n+1}-\nabla u_{k}^{n+1}\left(d_{k+1}^{n+1}-d_{k}^{n+1}\right), \quad \text { on } \quad \Gamma_{\text {trans }}^{\mathrm{w}} \text {, }
\end{aligned}
$$

The obtained fluid-subproblem allow us to take into account the interface motion, while keeping a fixed fluid domain. This is achieved by using non-standard boundary conditions on the fixed reference interface $\Gamma_{\text {trans }}^{\mathrm{w}}$, without the need of updating the mesh and, consequently, the system matrices.

Remark 4.1. In (13) the velocity $w_{k+1}^{n+1}$ is no longer needed inside the fluid domain. Therefore, we just compute it at the fluid structure interface $\Gamma_{0}^{\mathrm{w}}$.

In the same way, the fluid load at the moving interface can be recovered from a similar Taylor expansion:

$$
\sigma_{k+1}\left(x_{k+1}^{\mathrm{f}}\right)=\sigma_{k+1}\left(x_{k}^{\mathrm{f}}\right)+\nabla \sigma_{k+1}\left(x_{k}^{\mathrm{f}}\right)\left(x_{k+1}^{\mathrm{f}}-x_{k}^{\mathrm{f}}\right)+o\left(\left\|x_{k+1}^{\mathrm{f}}-x_{k}^{\mathrm{f}}\right\|\right)
$$


on $\Gamma_{0}^{\mathrm{w}}$. Thus, in (10), the solid subproblem can be now replaced by

$$
\begin{aligned}
& \frac{1}{\Delta t} m^{\mathrm{s}}\left(\dot{d}_{k+1}^{n+1}-\dot{d}^{n}, v^{\mathrm{s}}\right)+\frac{1}{2}\left(a^{\mathrm{s}}\left(d_{k}^{n+1}, v^{\mathrm{s}}\right)+a^{\mathrm{s}}\left(d^{n}, v^{\mathrm{s}}\right)\right) \\
& \quad=-\int_{\Gamma_{\mathrm{o}}^{\mathrm{w}}} J_{\mathcal{A}_{t_{n+1}}^{k+1}}\left(\sigma_{k+1}+\nabla \sigma_{k+1}\left(x_{k+1}^{\mathrm{f}}-x_{k}^{\mathrm{f}}\right)\right) F_{\mathcal{A}_{t_{n+1}}^{k+1}}^{-\mathrm{T}} n_{0} \cdot v^{\mathrm{s}} \mathrm{d} a, \quad \forall v^{\mathrm{s}} \in V^{\mathrm{s}}, \\
& \frac{1}{2}\left(\dot{d}_{k+1}^{n+1}+\dot{d}^{n}\right)=\frac{1}{\Delta t}\left(d_{k+1}^{n+1}-d^{n}\right), \quad \text { in } \Omega_{0}^{\mathrm{s}} .
\end{aligned}
$$

Remark 4.2. A simpler approximation, see $[6,16]$, can be obtained by replacing the first order Taylor expansions (11) and (14) by zeroth order expressions. Hence, the interface transpiration condition in (13) reduces to

$$
u_{k+1}^{n+1}=w_{k+1}^{n+1}, \quad \text { on } \quad \Gamma_{\text {trans }}^{\mathrm{w}},
$$

and the fluid interface load in (15) to

$$
\int_{\Gamma_{k}^{\mathrm{W}}} \sigma_{k+1} n \cdot v^{\mathrm{s}} \mathrm{d} a
$$

By exploiting the previous considerations we have derived the modified BGS algorithm reported in Figure 3. The boxes on the right of Figure 3 represents the transpiration loop. Here, instead of updating fluid mesh and matrices, we just enforce the transpiration velocity $w_{k+1}^{n+1}-\nabla u_{k}^{n+1}\left(x_{k+1}^{\mathrm{f}}-x_{k}^{\mathrm{f}}\right)$ at the interface. Tolerances $\mathrm{TOL}_{\text {trans }}^{\text {in }}$ and $\mathrm{TOL}_{\text {trans }}^{\text {out }}$ define the range of relative interface displacements where the transpiration formulation will be used. The convergence test of the whole algorithm is always made after two standard BGS iterations, ( $2 \mathrm{x}$ in the figure), in order to ensure the convergence of the original coupled problem. This also implies that the algorithm terminates with standard BGS iterations and with an updated mesh.

The convergence test can be done on the relative error

$$
\frac{\left\|d_{k+1}^{n+1}-d_{k}^{n+1}\right\|}{\left\|d_{k+1}^{n+1}\right\|}<\mathrm{TOL}
$$

in a suitable norm, for example the discrete $L^{\infty}$ norm. The main advantage of the relative error is that it is adimensional. However, when the structure is almost at rest, this stopping criterion becomes too restrictive. This is not the case when using adimensional absolute errors. Therefore, we have chosen here the following expression

$$
\frac{\left\|d_{k+1}^{n+1}-d_{k}^{n+1}\right\|}{\mathrm{d}^{\mathrm{ref}}}<\mathrm{TOL}
$$

where $\mathrm{d}^{\text {ref }}$ is a reference displacement for the fluid domain. For example, for the study of blood flow in an artery it could be the mean radius of the vessel.

In order to test whether to activate the transpiration part of the algorithm, the relative error described above is useless. Indeed, what we have to measure in this case is how much the computational fluid domain is distant from the actual fluid domain. The transpiration may be adopted only when this distance is small. Hence, the condition that has to be satisfied is

$$
\frac{\left\|d_{k+1}^{n+1}-d_{\text {trans }}^{n+1}\right\|}{L_{k}^{\text {ref }}}<\mathrm{TOL}_{\text {trans }}
$$

where $L_{k}^{\text {ref }}$ is a characteristic length of the fluid domain at the $k$ th iteration. In blood fluid dynamics $L_{k}^{\text {ref }}$ can be taken as the minimum of $\left\|R+d_{k}^{n+1}\right\|$ over the interface points. 


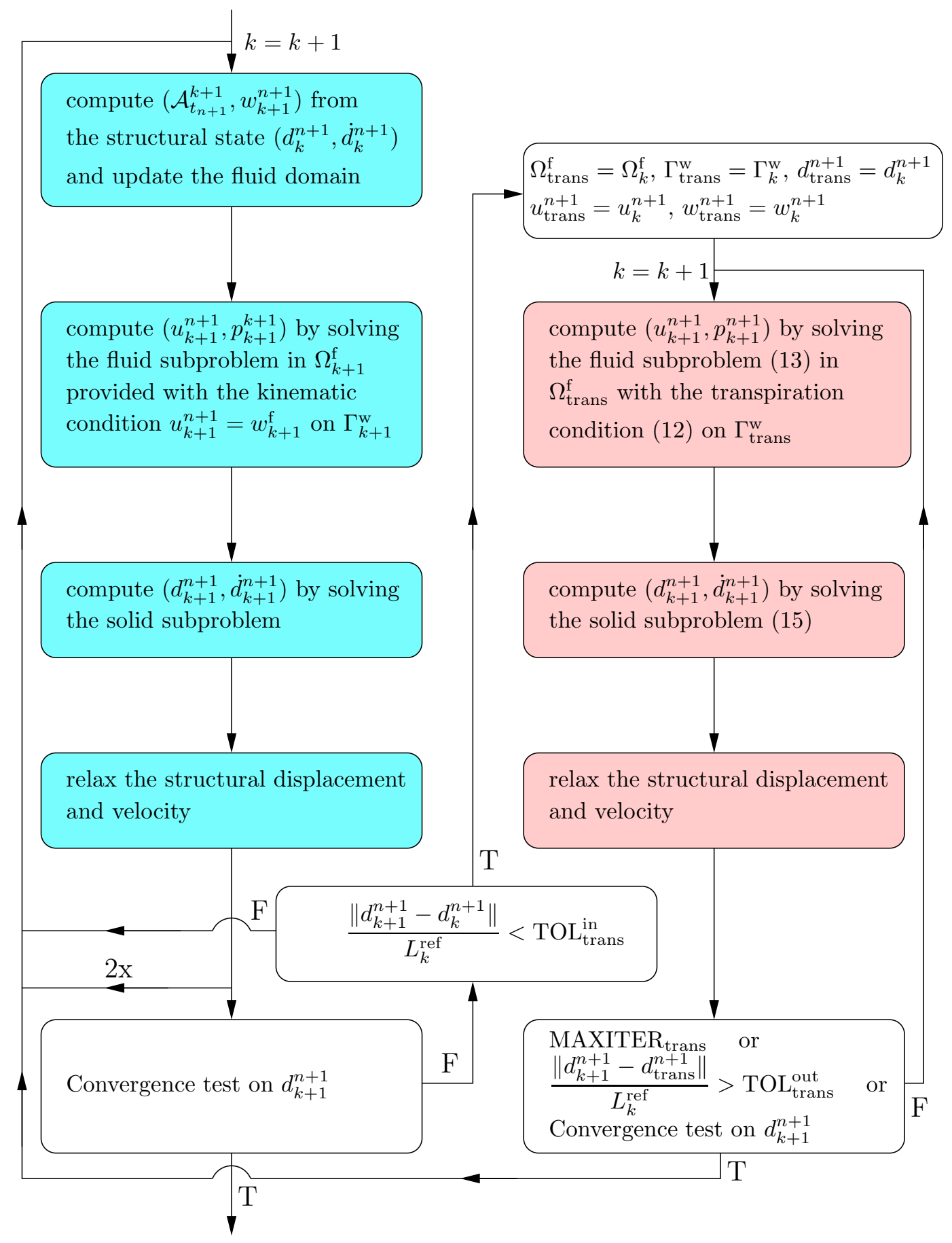

Figure 3. Diagram of the proposed algorithm. On the left, the BGS part; on the right, the transpiration steps. 


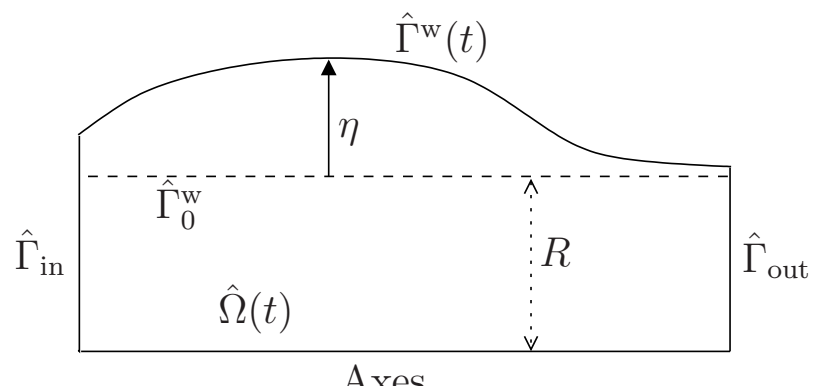

Figure 4. The computational domain.

\section{NumERICAL EXPERIMENT}

We have applied the above algorithm to a fluid-structure problem arising in the modeling of blood flow on large arteries. Namely, a thin elastic tube conveying an incompressible viscous fluid. In order to simplify the problem we considered the axisymmetric incompressible Navier-Stokes equations without rotation (see [4]) combined with a generalized string model (see [19]) for the structure. The initial domain is a cylinder of radius $R=0.5 \mathrm{~cm}$ and length $L=12 \mathrm{~cm}$ (see Fig. 4). The cylinder wall may deform only along the radial direction. The fluid and the structure are initially at rest.

The weak axisymmetric formulation of the Navier-Stokes equations reads

$$
\begin{aligned}
& \rho \frac{\mathrm{d}}{\mathrm{d} t} \int_{\hat{\Omega}(t)} r u \cdot v+\rho \int_{\hat{\Omega}(t)} r\left((u-w) \cdot \nabla_{a}\right) u \cdot v+\rho \int_{\hat{\Omega}(t)} r \operatorname{div}_{a}(w) u v+\rho \int_{\hat{\Omega}(t)} w_{r} u v+ \\
&+2 \mu \int_{\hat{\Omega}(t)} r \varepsilon_{a}(u): \varepsilon_{a}(v)+2 \mu \int_{\hat{\Omega}(t)} \frac{1}{r} u_{r} v_{r}-\int_{\hat{\Omega}(t)} r p \operatorname{div}_{a} v-\int_{\hat{\Omega}(t)} p v_{r} \\
& \quad+\int_{\hat{\Omega}(t)} r q \operatorname{div}_{a} u+\int_{\hat{\Omega}(t)} q u_{r}=\int_{\hat{\Omega}(t)} r f \cdot v+\int_{\hat{\Gamma}^{N}(t)} r g \cdot v,
\end{aligned}
$$

where

$$
u=\left(\begin{array}{c}
u_{r} \\
u_{z}
\end{array}\right), \quad w=\left(\begin{array}{c}
w_{r} \\
w_{z}
\end{array}\right), \quad v=\left(\begin{array}{c}
v_{r} \\
v_{z}
\end{array}\right), \quad f=\left(\begin{array}{c}
f_{r} \\
f_{z}
\end{array}\right), \quad g=\left(\begin{array}{c}
g_{r} \\
g_{z}
\end{array}\right)
$$

and

$$
\varepsilon_{a}(u)=\frac{1}{2}\left(\nabla_{a} u+\left(\nabla_{a} u\right)^{T}\right), \quad \nabla_{a}=\left(\begin{array}{c}
\partial_{r} \\
\partial_{z}
\end{array}\right), \quad \operatorname{div}_{a} u=\partial_{r} u_{r}+\partial_{z} u_{z}
$$

stand for differential operators in the cylindrical coordinates, $\hat{\Omega}(t)$ for the half section of the fluid domain $\Omega^{\mathrm{f}}(t)$ at time $t$ and $\hat{\Gamma}^{N}(t)=\hat{\Gamma}_{\text {in }}(t) \cup \hat{\Gamma}_{\text {out }}(t)$ the inlet and outlet Neumann boundary sections.

The wall displacement $\eta$ is modeled by a vibrating string model (see [22]) governed by the equation

$$
\rho_{w} h \frac{\partial^{2} \eta}{\partial t^{2}}-S_{z} \frac{\partial^{2} \eta}{\partial z^{2}}+\frac{E h}{\left(1-\nu^{2}\right) R^{2}} \eta=H
$$

where the forcing term $H$ is equal to $\left(\sigma\left(u, p-p_{0}\right) n\right) \cdot e_{r}$ (the full contribution of the fluid Cauchy tensor to the normal stress is taken into account). The external pressure $p_{0}$ and the initial pressure of the fluid are both set equal to zero. The wall density is $\rho_{w}=1.1 \mathrm{~g} / \mathrm{cm}^{3}$, its thickness $h=0.1 \mathrm{~cm}$, Young Modulus $E=0.75 \cdot 10^{4}$ dyne $/ \mathrm{cm}^{2}$, Poisson coefficient $\nu=0.5$ and longitudinal stress $S_{z}=2.5 \cdot 10^{4}$ dyne. 


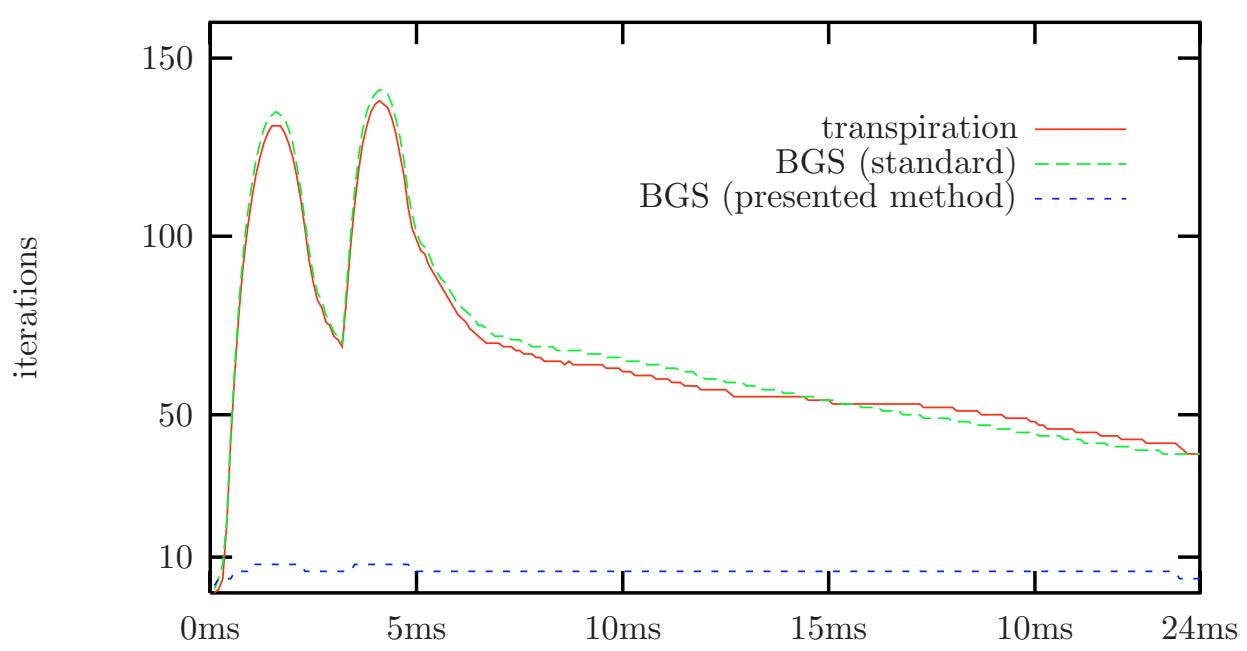

FIGURE 5. Iterations history.

On the outlet we impose $\sigma(u, p) n=0$ and on the inlet one "pressure wave" of a period of $5 \mathrm{~ms}$, i.e.,

$$
\sigma(u, p) n= \begin{cases}-\frac{P_{\text {in }}}{2}\left[1-\cos \left(\frac{2 \pi t}{5}\right)\right] n, & t \leq 5 \mathrm{~ms} \\ 0, & t>5 \mathrm{~ms}\end{cases}
$$

with $P_{\text {in }}=2 \cdot 10^{4}$ dyne $/ \mathrm{cm}^{2}$. The fluid density is $\rho=1 \mathrm{~g} / \mathrm{cm}^{3}$ and the viscosity $\mu=0.035$ poise. We have adopted axisymmetric $\mathbb{P}_{1}$ iso $\mathbb{P}_{2} / \mathbb{P}_{1}$ finite elements for the fluid and $\mathbb{P}_{1}$ for the structure. The time is discretized by a mid-point scheme for the structure and implicit Euler for the fluid equations (see [19]), with a time step of $\Delta t=0.1 \mathrm{~ms}$ and a relaxation parameter $\omega=0.01$.

We have used the simplified form (16) for the forcing term and the following values for the tolerances in the proposed numerical scheme: TOL $=10^{-6}$ for the fixed-point (absolute) convergence test with reference displacement equal to $10 \%$ of the initial radius of the artery, $\mathrm{TOL}_{\text {trans }}^{\text {in }}=0.05, \mathrm{TOL}_{\text {trans }}^{\text {out }}=0.1$ and $\mathrm{MAXITER}_{\text {trans }}=50$. We take as characteristic length of the domain the initial radius of the artery, $L_{k}^{\text {ref }}=R$.

In Figure 5 we report the number of sub-iterations per time step required by the standard BGS method compared with the one obtained using the modified BGS scheme with transpiration. The number of BGS iterations is strongly reduced in the transpiration version. Let us notice that at each time step, the number of outer iterations is almost equal in the two schemes. However, the computing time is greatly reduced: a gain of $40 \%$ over 240 time steps. Figure 6 shows the wall displacement obtained by the standard and accelerated BGS method at three different time steps. This figure points out that the proposed algorithm does not introduce loss of accuracy. Indeed, as mentioned above, the converged solution provided by our algorithm is also an iteration of the standard BGS method. Finally, in Figure 7 we plot the corresponding fluid pressure.

We have also tested the zeroth order formulation with (12) substituted by $u_{k+1}^{n+1}=w_{k+1}^{n+1}$ (see Rem. 4.2). The CPU time and the number of iterations are of the same order (see Tab. 1). The slight difference in CPU time derives from the computation of the fluid velocity gradients. The fact that the convergence obtained with the two alternatives is similar is due to the limited contribution of the velocity gradients for this test case. Indeed, the additional contribution given by the first order scheme is only $10^{-7}$ times the zeroth order term. This hangs on from the very little variations in the wall displacement between the first two BGS iterations and the following transpiration ones. 


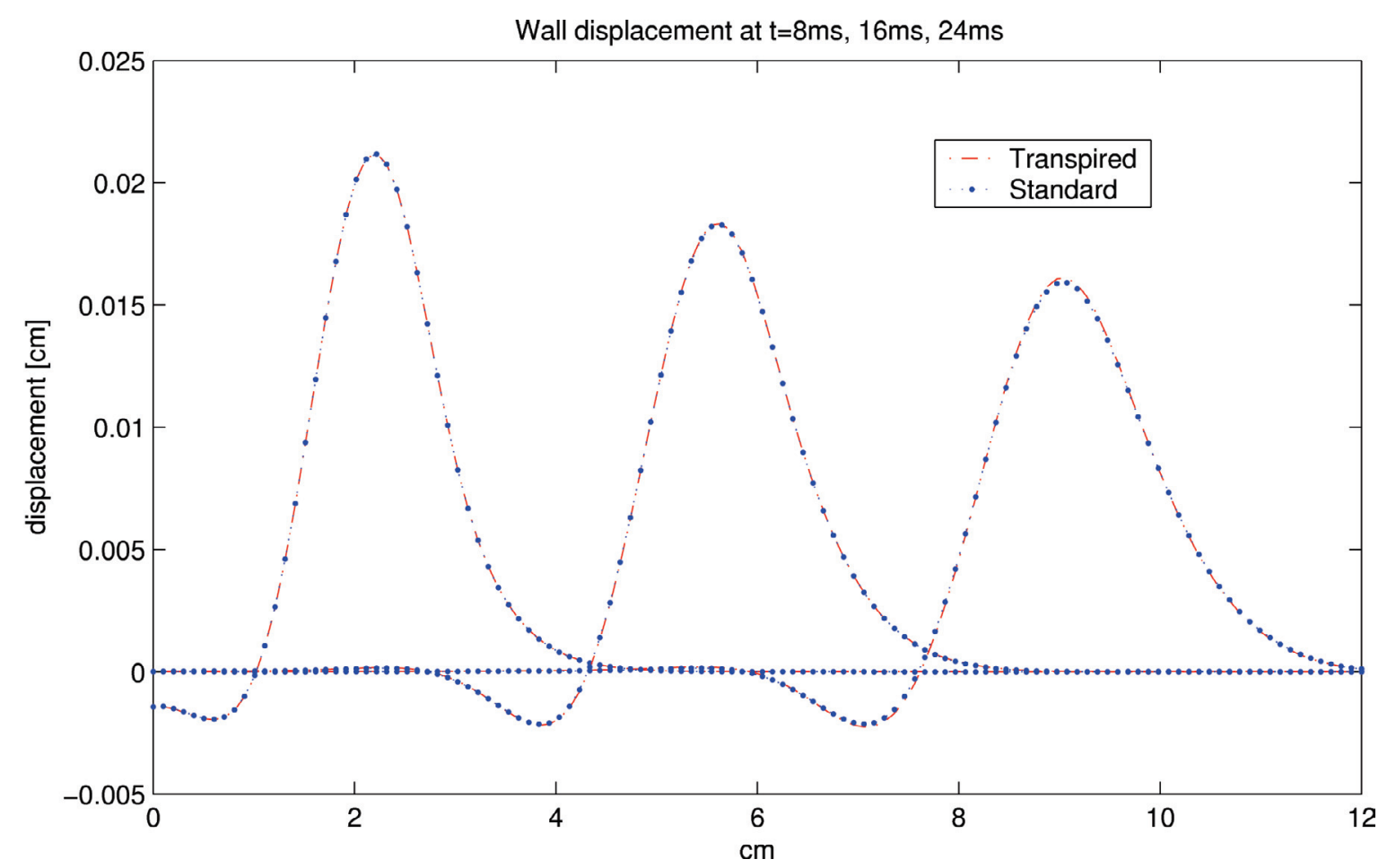

Figure 6. Wall displacement.

TABLE 1. CPU time w.r.t. standard BGS or first order transpiration scheme.

\begin{tabular}{|c|c|c|}
\hline MAXITER $_{\text {trans }}$ & 50 & 200 \\
\hline Standard & \multicolumn{2}{|c|}{$113 \mathrm{~min}$} \\
\hline Order 0 & $66 \mathrm{~min}$ & $67 \mathrm{~min}$ \\
\hline Order 1 & $69 \mathrm{~min}$ & $72 \mathrm{~min}$ \\
\hline
\end{tabular}

Remark 5.1. The superiority of the first order transpiration condition with respect to the zeroth one can be easily underlined when the difference between the transpiration domain and the actual fluid domain are more significant. For example one can modify the algorithm in Figure 3 in order to update the fluid mesh only at the beginning of the time step or every $n$ time steps (see Fig. 8).

\section{Conclusion}

In this work we dealt with the numerical solution of fluid-structure interaction problems in which the fluid density is comparable to that of the structure (for instance in hemodynamics applications). Thus, at each time step, we had to solve a highly non-linear coupled system, since the fluid domain depends on the unknown structural motion. We proposed a modified fixed-point algorithm which combines the Block-Gauss-Seidel iterations with a transpiration formulation. Numerical experiments pointed out the relevant improvement of computing time with respect to the standard method.

The sensibility analysis of the different parameters MAXITER $_{\text {trans }}$, TOL, use of zeroth or first order transpiration formulation) as well as variants of the algorithm and other tests will be reported in a forthcoming work (see [4]). 
Pressure Wave $($ time $=8 \mathrm{~ms})$

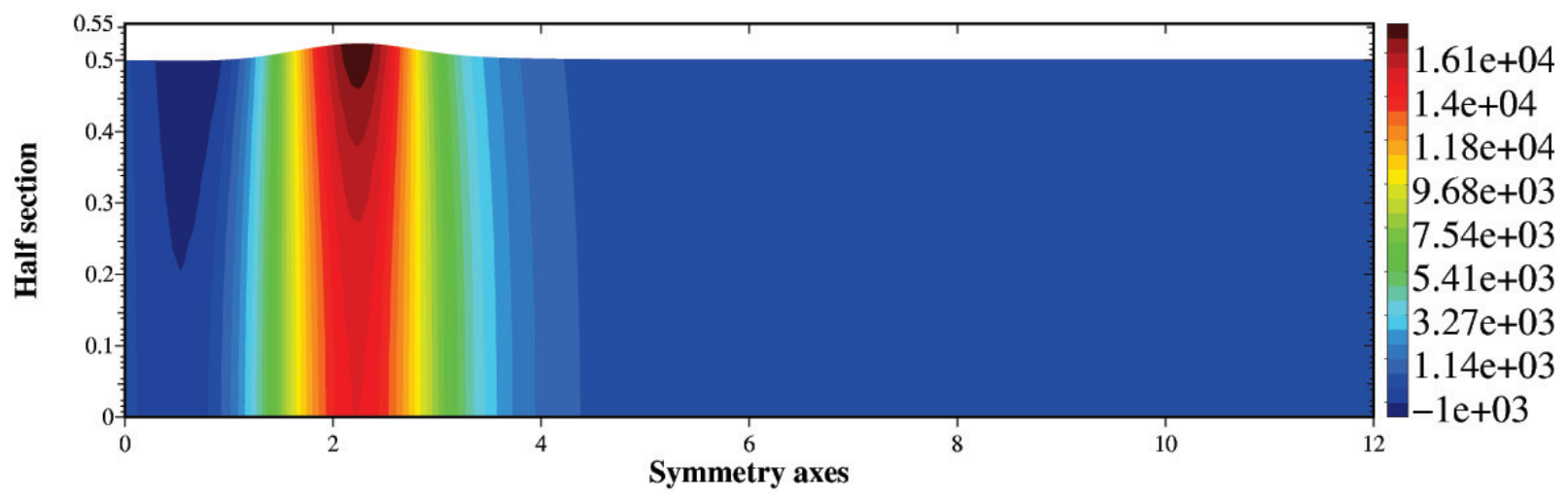

Pressure Wave $($ time $=16 \mathrm{~ms})$

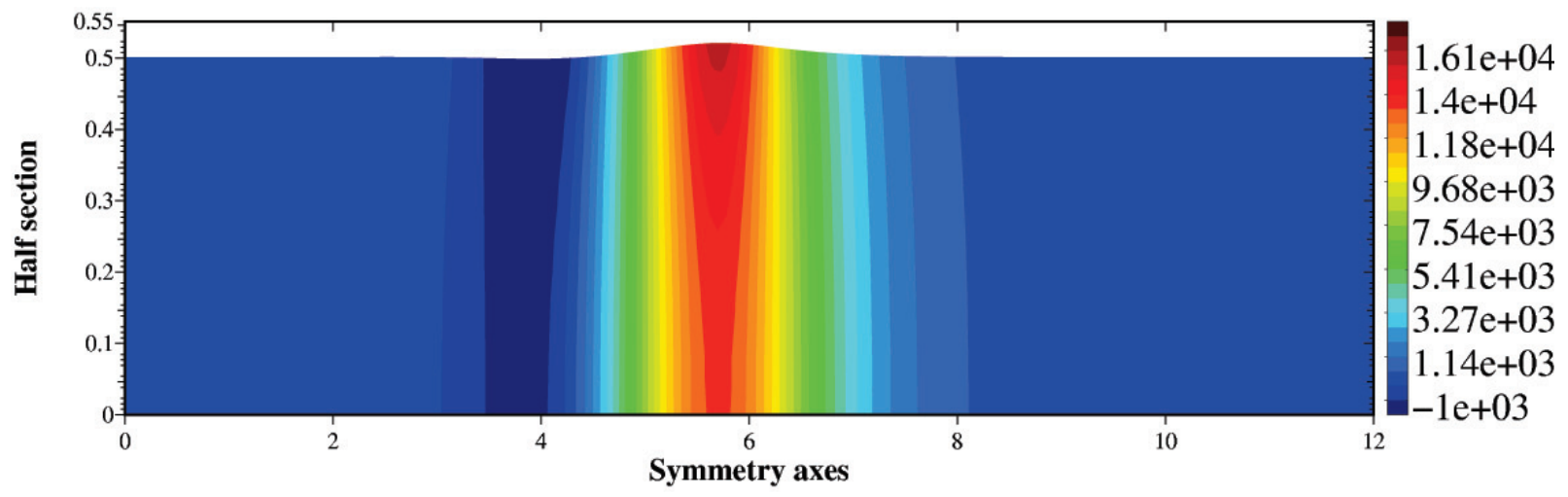

Pressure Wave $($ time $=24 \mathrm{~ms})$

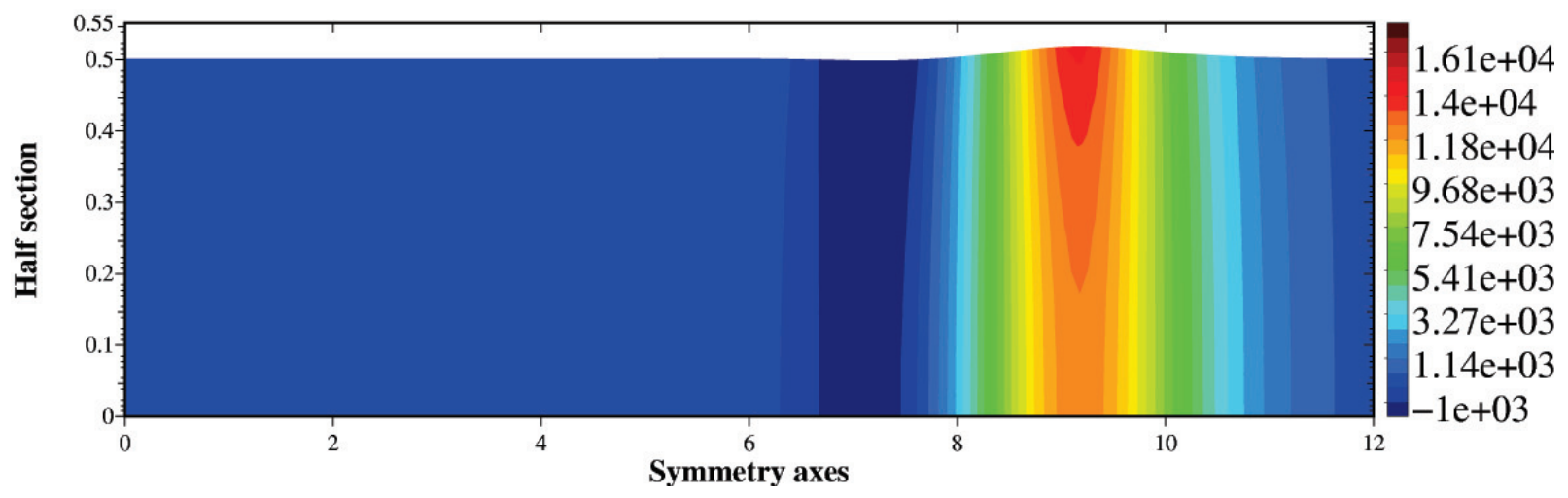

Figure 7. Pressure wave. 


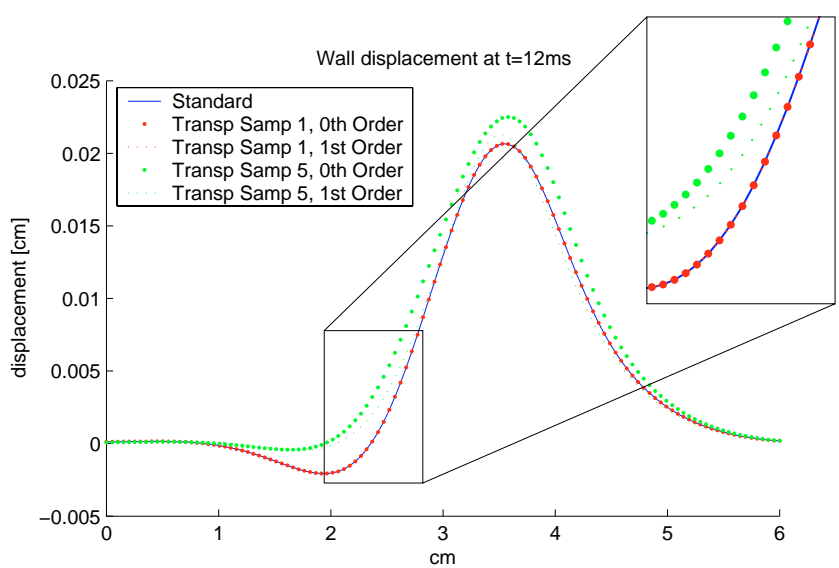

Figure 8. Comparison of the zeroth and first order transpiration condition. The domain is updated only at the beginning of the BGS iterations (Samp 1) or every 5 time steps (Samp 5). We have zoomed a portion of the picture to better evidentiate the different curves. The enhanced accuracy a the second order transpiration condition is appreciated when the domain is updated less often.

Acknowledgements. The first author was supported by the ETH board through a stipend for exchange between ETH Zürich and EPF Lausanne. The second author was supported by the Swiss National Science Foundation (contract number 20-65110.01). The third author acknowledges partial support by the European Union through the RTN project HaeMOdel.

\section{REFERENCES}

[1] M. Carrive-Bédouani, P. Le Tallec and J. Mouro, Approximation par éléments finis d'un modèle de coques minces géométriquement exact. Rev. Européenne Élém. Finis 4 (1995) 633-661.

[2] P.G. Ciarlet, Mathematical Elasticity, Vol. I, Three-Dimensional Elasticity. North-Holland, Amsterdam (1988).

[3] R. Codina and M. Cervera, Block Iterative Algorithms for Non-Linear Coupled Problems. Advanced Computational Methods in Structural Mechanics, CIMNE, Barcelona (1996).

[4] S. Deparis, Fluid-Structure Interaction Problems in Hemodynamics and Axisymmetric Modeling of Blood Flow. Ph.D. thesis, École Politechnique Fédérale de Lausanne (EPFL), in preparation.

[5] J. Donea, An Arbitrary Lagrangian-Eulerian Finite Element Method for Transient Dynamic Fluid-Structure Interactions. Comput. Methods Appl. Mech. Engrg. 33 (1982) 689-723.

[6] T. Fanion, M.A. Fernández and P. Le Tallec, Deriving Adequate Formulations for Fluide Structure Interaction Problems: from ALE to Transpiration. Rev. Européenne Élém. Finis 9 (2000) 681-708.

[7] C. Farhat and M. Lesoinne, Two Efficient Staggered Algorithms for the Serial and Parallel Solution of Three-Dimensional Nonlinear Transient Aeroelastic Problems. Comput. Methods Appl. Mech. Engrg. 182 (2000) 499-515.

[8] M.A. Fernández, Modèles Simplifiés d'Interaction Fluide-Structure. Ph.D. thesis, University of Paris IX, France (2001).

[9] M.A. Fernández and M. Moubachir, An Exact Block-Newton Algorithm for the Solution of Implicit Time Discretized Coupled Systems Involved in Fluid-Structure Interaction Problems, in Second MIT Conference on Computational Fluid and Solid Mechanics, Elsevier (2003).

[10] L. Formaggia and F. Nobile, A Stability Analysis for the Arbitrary Lagrangian Eulerian Formulation with Finite Elements. East-West J. Numer. Math. 7 (1999) 105-131.

[11] J.F. Gerbeau and M. Vidrascu, A Quasi-Newton Algorithm Based on a Reduced Model for Fluid Structure Problems in Blood Flows. ESAIM: M2AN 37 (2003) 631-647.

[12] W.P. Huffman, R.G. Melvin, D.P. Young, F.T. Johnson, J.E. Bussoletti, M.B. Bieterman and C.L. Hilmes, Practical Design and Optimisation in Computational Fluids Dynamics, in proceedings of the AIAA 24th Fluid Dynamics Conference, Orlando, Florida (1993) 93-3111.

[13] P. Le Tallec, Numerical Methods for Nonlinear Three-Dimensional Elasticity. Handbook of Numerical Analysis, Vol. III, North-Holland, Amsterdam (1994) 465-622. 
[14] P. Le Tallec and J. Mouro, Fluid Structure Interaction with Large Structural Displacements. Comput. Methods Appl. Mech. Engrg. 190 (2001) 3039-3067.

[15] M.J. Lighthill, On Displacement Thickness. J. Comput. Phys. 4 (1958) 383-392.

[16] G. Medic, Étude mathématique des modèles aux tensions de Reynolds et simulation numérique d'écoulements turbulents sur parois fixes et mobiles. Ph.D. thesis, University of Paris VI, France (1999).

[17] D.P. Mok, W.A. Wall and E. Ramm, Accelerated Iterative Substructuring Schemes for Instationnary Fluid-structure Interaction, in proceedings of the First MIT Conference on Computational Fluid and Solid Mechanics, Elsevier (2001) $1325-1328$.

[18] M. Moubachir, Mathematical and Numerical Analysis of Inverse and Control Problems for Fluid-Structure Interaction Systems. Ph.D. thesis, École Nationale des Ponts et Chaussées, France (2002).

[19] F. Nobile, Numerical Approximation of Fluid-Structure Interaction Problems with Application to Haemodynamics. Ph.D. thesis, École Polytechnique Fédérale de Lausanne, Switzerland (2001).

[20] S. Piperno, C. Farhat and B. Larrouturou, Partitioned Procedures for the Transient Solution of Coupled Aeroelastic Problems Part I: Model Problem, Theory and Two-Dimensional Application. Comput. Methods Appl. Mech. Engrg. 124 (1995) 79-112.

[21] S. Piperno, C. Farhat and B. Larrouturou, Partitioned Procedures for the Transient Solution of Coupled Aeroelastic Problems Part II: Energy Transfer Analysis and Three-Dimensional applications. Comput. Methods Appl. Mech. Engrg. 124 (1995) 79112 .

[22] A. Quarteroni and L. Formaggia, Mathematical Modelling and Numerical Simulation of the Cardiovascular System. To appear as chapter in Modelling of Living Systems, N. Ayache Ed., Handbook of numerical Analysis Series, Elsevier, Amsterdam (2003).

[23] P. Raj and B. Harris, Using Surface Transpiration With an Euler Method for Cost-Effective Aerodynamic Analysis, in proceedings of the AIAA 24th Applied Aerodynamics Conference, 93-3506, Monterey, Canada (1993).

[24] J.Y. Renou, Une méthode eulérienne pour le calcul de forces fluide-élastiques. Ph.D. thesis, University of Paris VI, France (1998).

[25] J. Steindorf and H.G Matthies, Numerical Efficiency of Different Partitioned Methods for Fluid-Structure interaction. ZAMM, Z. Angew. Math. Mech. 80 (2000) 557-558.

To access this journal online:

www.edpsciences.org 